DESY 95-210

KA-TP-8-95

hep-ph/9511344

November 1995

\title{
QCD Corrections to Hadronic Higgs Decays
}

\author{
A. DJoundi ${ }^{1,2}$ 丹, M. Spira ${ }^{3}$ And P.M. Zerwas ${ }^{2}$ \\ ${ }^{1}$ Institut für Theoretische Physik, Universität Karlsruhe, \\ D-76128 Karlsruhe, FRG. \\ ${ }^{2}$ Deutsches Elektronen-Synchrotron DESY, D-22603 Hamburg, FRG. \\ ${ }^{3}$ II. Institut für Theoretische Physik|, D-22761 Hamburg, FRG.
}

\begin{abstract}
We present an update of the branching ratios for Higgs decays in the Standard Model and the Minimal Supersymmetric extension of the Standard Model. In particular, the decays of the Higgs particles to quark and gluon jets are analyzed and the spread in the theoretical predictions due to uncertainties of the quark masses and the QCD coupling is discussed.
\end{abstract}

\footnotetext{
*Supported by Deutsche Forschungsgemeinschaft DFG (Bonn).

${ }^{\dagger}$ Supported by Bundesministerium für Bildung und Forschung (BMBF), Bonn, under Contract 056 HH 93P (5), and by EU Program Human Capital and Mobility through Network Physics at High Energy Colliders under Contract CHRX-CT93-0357 (DG12 COMA).
} 


\section{Introduction}

The coupling of the Higgs bosons to other particles grows with the mass of the particles. This characteristic property is a direct consequence of mass generation through the Higgs mechanism. To establish the Higgs mechanism experimentally, it is therefore mandatory to measure the couplings very accurately [1] once scalar particles have been found. The main test grounds for the Higgs couplings to gauge bosons are the production cross sections for Higgs-strahlung off gauge bosons and $W W / Z Z$ fusion, and the widths/branching ratios for Higgs decays to gauge bosons. The Higgs couplings to heavy quarks determine the cross sections for the production of Higgs particles in $g g$ fusion at hadron colliders [2, 3], as well as the rate of Higgs bremsstrahlung off heavy quarks at $e^{+} e^{-}$[4] and hadron colliders [5]. The measurement of Higgs decay branching ratios, including $b, c$ quarks and $\tau$ leptons [6], provides a complementary method to determine the Higgs couplings.

In this note we will reanalyze [7] the branching ratios for Higgs decays to $b, c$ quark jets and to light hadron jets evolving out of gluon decays,

$$
\begin{aligned}
& H \rightarrow b \bar{b} / c \bar{c}+\ldots \\
& H \rightarrow g g+\ldots
\end{aligned}
$$

The ellipses indicate additional gluon and quark partons in the final state due to QCD radiative corrections. Special attention will be paid to uncertainties related to the $b, c$ quark masses and the QCD coupling $\alpha_{s}$. It turns out that the evolution of the charm quark mass from low energy scales, where it can be determined by QCD sum rules, to high energy scales defined by the Higgs mass, introduces very large uncertainties in the $c \bar{c}$ branching ratio. The partial width of the second decay mode (2) will be derived for gluon and light quark final states since heavy quarks add to the partial width of the first decay process (1). The $b, c$ and gluon decay modes are experimentally important in the Standard Model $(\mathcal{S M})$ for Higgs masses less than about $150 \mathrm{GeV}$. In the minimal supersymmetric extension $(\mathcal{M S S M}) b$ quark decays may be dominant for a much wider range in the parameter space.

\section{Standard Model}

\section{$2.1 \mathrm{~b}$, c quark decays of the $\mathcal{S M}$ Higgs particle}

The particle width for decays to (massless) $b, c$ quarks directly coupled to the $\mathcal{S} \mathcal{M}$ Higgs particle is given, up to $\mathcal{O}\left(\alpha_{s}^{2}\right)$ QCD radiative corrections expression [8, 9, 10]

$$
\Gamma[H \rightarrow Q \bar{Q}]=\frac{3 G_{F} M_{H}}{4 \sqrt{2} \pi} \bar{m}_{Q}^{2}\left(M_{H}\right)\left[\Delta_{\mathrm{QCD}}+\Delta_{t}\right]
$$

\footnotetext{
${ }^{1}$ The effect of the electroweak radiative corrections in the branching ratios is negligible [1].
} 


$$
\begin{aligned}
\Delta_{\mathrm{QCD}} & =1+5.67 \frac{\alpha_{s}\left(M_{H}\right)}{\pi}+\left(35.94-1.36 N_{F}\right)\left(\frac{\alpha_{s}\left(M_{H}\right)}{\pi}\right)^{2} \\
\Delta_{t} & =\left(\frac{\alpha_{s}\left(M_{H}\right)}{\pi}\right)^{2}\left[1.57-\frac{2}{3} \log \frac{M_{H}^{2}}{M_{t}^{2}}+\frac{1}{9} \log ^{2} \frac{\bar{m}_{Q}^{2}\left(M_{H}\right)}{M_{H}^{2}}\right]
\end{aligned}
$$

in the $\overline{\mathrm{MS}}$ renormalization scheme; the running quark mass and the QCD coupling are defined at the scale of the Higgs mass, absorbing this way any large logarithms. The quark masses can be neglected in general except for top quark decays where this approximation holds only sufficiently far above threshold; the QCD correction in this case are given in the Appendix.

Since the relation between the pole mass $M_{c}$ of the charm quark and the $\overline{\mathrm{MS}}$ mass evaluated at the pole mass $\bar{m}_{c}\left(M_{c}\right)$ is badly convergent [12], we will adopt the running quark masses $\bar{m}_{Q}\left(M_{Q}\right)$ as starting points. They have been extracted directly from QCD sum rules evaluated in a consistent $\mathcal{O}\left(\alpha_{s}\right)$ expansion [13]. The evolution from $M_{Q}$ upwards to a renormalization scale $\mu$ is given by

$$
\bar{m}_{Q}(\mu)=\bar{m}_{Q}\left(M_{Q}\right) \frac{c\left[\alpha_{s}(\mu) / \pi\right]}{c\left[\alpha_{s}\left(M_{Q}\right) / \pi\right]}
$$

with [9, 12]

$$
\begin{array}{ll}
c(x)=\left(\frac{25}{6} x\right)^{\frac{12}{25}}\left[1+1.014 x+1.389 x^{2}\right] & \text { for } M_{c}<\mu<M_{b} \\
c(x)=\left(\frac{23}{6} x\right)^{\frac{12}{23}}\left[1+1.175 x+1.501 x^{2}\right] & \text { for } M_{b}<\mu
\end{array}
$$

For the charm quark mass the evolution is determined by eq.(4) up to the scale $\mu=M_{b}$, while for scales above the bottom mass the evolution must be restarted at $M_{Q}=M_{b}$. Typical values of the running $b, c$ masses at the scale $\mu=100 \mathrm{GeV}$, characteristic for the Higgs mass, are displayed in Table 1. The evolution has been calculated for the QCD coupling

$$
\alpha_{s}\left(M_{Z}\right)=0.118 \pm 0.006
$$

defined at the $Z$ mass [14. The large uncertainty in the running charm mass is a consequence of the small scale at which the evolution starts and where the errors of the QCD coupling are very large.

\subsection{Higgs decay to light hadron jets}

The decay of the Higgs boson to gluons is mediated by heavy quark loops in the Standard Model (Fig.1b); the partial decay width [15] is given by

$$
\Gamma_{L O}[H \rightarrow g g]=\frac{G_{F} \alpha_{s}^{2} M_{H}^{3}}{36 \sqrt{2} \pi^{3}}\left|\sum_{t, b} A^{H}\left(\tau_{Q}\right)\right|^{2}
$$




\begin{tabular}{|c|c|cc|c|}
\hline & $\alpha_{s}\left(M_{Z}\right)$ & $\bar{m}_{Q}\left(M_{Q}\right)$ & $M_{Q}=M_{Q}^{p t 2}$ & $\bar{m}_{Q}(\mu=100 \mathrm{GeV})$ \\
\hline \multirow{2}{*}{$b$} & 0.112 & $(4.26 \pm 0.02) \mathrm{GeV}$ & $(4.62 \pm 0.02) \mathrm{GeV}$ & $(3.04 \pm 0.02) \mathrm{GeV}$ \\
& 0.118 & $(4.23 \pm 0.02) \mathrm{GeV}$ & $(4.62 \pm 0.02) \mathrm{GeV}$ & $\begin{array}{l}(2.92 \pm 0.02) \mathrm{GeV} \\
(2.80 \pm 0.02) \mathrm{GeV}\end{array}$ \\
& 0.124 & $(4.19 \pm 0.02) \mathrm{GeV}$ & $(4.62 \pm 0.02) \mathrm{GeV}$ & \\
\hline \multirow{2}{*}{$c$} & & & & \\
& 0.112 & $(1.25 \pm 0.03) \mathrm{GeV}$ & $(1.42 \pm 0.03) \mathrm{GeV}$ & $(0.69 \pm 0.02) \mathrm{GeV}$ \\
& 0.118 & $(1.23 \pm 0.03) \mathrm{GeV}$ & $(1.42 \pm 0.03) \mathrm{GeV}$ & $(0.62 \pm 0.02) \mathrm{GeV}$ \\
& 0.124 & $(1.19 \pm 0.03) \mathrm{GeV}$ & $(1.42 \pm 0.03) \mathrm{GeV}$ & $(0.53 \pm 0.02) \mathrm{GeV}$ \\
& & & & \\
\hline
\end{tabular}

Table 1: The running b,c quark masses in the $\overline{\mathrm{MS}}$ renormalization scheme at the scale $\mu=100 \mathrm{GeV}$. The starting points $\bar{m}_{Q}\left(M_{Q}\right)$ of the evolution are extracted from $Q C D$ sum rules [13]; the pole masses $M_{Q}^{p t 2}$ are defined by the $\mathcal{O}\left(\alpha_{s}\right)$ relation $\bar{m}_{Q}\left(M_{Q}^{p t 2}\right)=$ $M_{Q}^{p t 2} /\left[1+4 \alpha_{s} / 3 \pi\right]$ with the running masses.

with the form factor

$$
\begin{array}{rlr}
A^{H}(\tau) & =\frac{3}{2} \tau[1+(1-\tau) f(\tau)] \\
f(\tau) & = \begin{cases}\arcsin ^{2} \frac{1}{\sqrt{\tau}} & \tau \geq 1 \\
-\frac{1}{4}\left[\log \frac{1+\sqrt{1-\tau}}{1-\sqrt{1-\tau}}-i \pi\right]^{2} & \tau<1\end{cases}
\end{array}
$$

The parameter $\tau_{Q}=4 M_{Q}^{2} / M_{H}^{2}$ is defined by the pole mass of the heavy loop quark $Q$. For large quark masses the form factor approaches unity. QCD radiative corrections are built up by the exchange of virtual gluons and the splitting of a gluon into two gluons or a quark-antiquark pair, Fig.1b. If all quarks $u, \cdots, b$ are treated massless at the renormalization scale $\mu \sim M_{H} \sim 100 \mathrm{GeV}$, the radiative corrections can be approximated very well by [16]

$$
\begin{gathered}
\Gamma^{N_{F}}[H \rightarrow g g(g), q \bar{q} g]=\Gamma_{L O}\left[\alpha_{s}^{\left(N_{F}\right)}(\mu)\right]\left\{1+E^{N_{F}} \frac{\alpha_{s}^{\left(N_{F}\right)}(\mu)}{\pi}\right\} \\
E^{N_{F}}=\frac{95}{4}-\frac{7}{6} N_{F}+\frac{33-2 N_{F}}{6} \log \frac{\mu^{2}}{M_{H}^{2}}
\end{gathered}
$$

with $N_{F}=5$ light quark flavors. The radiative corrections are very large, nearly doubling the partial width. 
The final states $H \rightarrow b \bar{b} g$ and $c \bar{c} g$ are also generated through processes in which the $b, c$ quarks are coupled to the Higgs boson directly (Fig. 1a). Gluon splitting $g \rightarrow b \bar{b}$ in $H \rightarrow g g$ (Fig. 1b) increases the inclusive decay probabilities $[\tilde{f}(H \rightarrow b \bar{b}+\ldots)$ etc. Since $b$ quarks, and eventually c quarks, can in principle be tagged experimentally, it is physically meaningful to consider the particle width of Higgs decays to gluon and light $u, d, s$ quark final jets separately. The contribution of $b, c$ quark final states to the coefficient $E^{N_{F}}$ in eq.(17) is given by

$$
\delta E^{b, c}=-\frac{7}{3}+\frac{1}{3}\left[\log \frac{M_{H}^{2}}{M_{b}^{2}}+\log \frac{M_{H}^{2}}{M_{c}^{2}}\right]
$$

in the limit $M_{H}^{2} \gg M_{b, c}^{2}$. Instead of naively subtracting this contribution, it may be noticed that the mass logarithms can be absorbed by changing the number of active flavors from $N_{F}=5$ to $N_{L}=3$ in the QCD coupling,

$$
\alpha_{s}^{(5)}(\mu)=\alpha_{s}^{(3)}(\mu)\left\{1+\frac{\alpha_{s}^{(3)}}{6 \pi}\left[\log \frac{\mu^{2}}{M_{c}^{2}}+\log \frac{\mu^{2}}{M_{b}^{2}}\right]+\ldots\right\}
$$

This way we arrive again at an equally simple expression

$$
\begin{gathered}
\Gamma[H \rightarrow g g(g), q \bar{q} g]=\Gamma_{L O}\left[\alpha_{s}^{\left(N_{L}\right)}(\mu)\right]\left\{1+E^{N_{L}} \frac{\alpha_{s}^{\left(N_{L}\right)}(\mu)}{\pi}\right\} \\
E^{N_{L}}=\frac{95}{4}-\frac{7}{6} N_{L}+\frac{33-2 N_{L}}{6} \log \frac{\mu^{2}}{M_{H}^{2}}
\end{gathered}
$$

for $N_{L}=3$ light $q=u, d, s$ quark flavors in the final state.

The subtracted parts may be added to the partial decay widths into $c$ and $b$ quarks. Up to $\mathcal{O}\left(\alpha_{s}^{3}\right)$, they are given by the difference of the gluonic widths [eq.(7))] for the corresponding number of flavors $N_{F}$,

$$
\begin{aligned}
& \delta \Gamma[H \rightarrow c \bar{c}+\ldots]=\Gamma^{4}-\Gamma^{3} \\
& \delta \Gamma[H \rightarrow b \bar{b}+\ldots]=\Gamma^{5}-\Gamma^{4}
\end{aligned}
$$

The $\overline{\mathrm{MS}} \Lambda$ parameters for three, four and five flavors in the QCD coupling

$$
\alpha_{s}^{\left(N_{F}\right)}(\mu)=\frac{12 \pi}{\left(33-2 N_{F}\right) \log \left(\mu^{2} / \Lambda_{N_{F}}^{2}\right)}\left[1-6 \frac{153-19 N_{F}}{\left(33-2 N_{F}\right)^{2}} \frac{\log \log \left(\mu^{2} / \Lambda_{N_{F}}^{2}\right)}{\log \left(\mu^{2} / \Lambda_{N_{F}}^{2}\right)}\right]
$$

are given in Table 2 together with the effective couplings $\alpha_{s}^{\left(N_{F}\right)}\left(M_{Z}\right)$ for three, four and five flavors corresponding to the QCD scales $\Lambda_{N_{F}}$. In $\alpha_{s}^{(4)}\left(M_{Z}\right)$ the contribution of the $b$ quark is subtracted and in $\alpha_{s}^{(3)}\left(M_{Z}\right)$ the contributions of both the $b$ and $c$ quarks.

\footnotetext{
${ }^{2}$ The two contributions add up incoherently in the limit where the final state quark masses are neglected [apart from the Yukawa Higgs couplings]. The topology of the final states is in general different for decays to $b, c$ quarks through the direct couplings or gluon splitting: in the former decay mode, quark and anti-quark jets are emitted primarily back-to-back, while in the latter decay mode they tend to be collinear.
} 


\begin{tabular}{|c|c||c|c||c|c|}
\hline$\alpha_{s}^{(5)}\left(M_{Z}\right)$ & $\Lambda_{5}[\mathrm{MeV}]$ & $\alpha_{s}^{(4)}\left(M_{Z}\right)$ & $\Lambda_{4}[\mathrm{MeV}]$ & $\alpha_{s}^{(3)}\left(M_{Z}\right)$ & $\Lambda_{3}[\mathrm{MeV}]$ \\
\hline \hline & & & & & \\
0.112 & 159 & 0.107 & 238 & 0.101 & 286 \\
0.118 & 226 & 0.113 & 327 & 0.105 & 378 \\
0.124 & 312 & 0.118 & 434 & 0.110 & 483 \\
\hline
\end{tabular}

Table 2: QCD scales $\Lambda_{N_{F}}$ for the range of uncertainty in the strong coupling constant $\alpha_{s}^{(5)}\left(M_{Z}\right)=0.118 \pm 0.006$. The effective couplings $\alpha_{s}^{(4)}\left(M_{Z}\right)$, with the $b$ quark decoupled, and $\alpha_{s}^{(3)}\left(M_{Z}\right)$, with both the $b$ and $c$ quarks decoupled, are given for comparison, too.

With $E^{3}=20.25$, the QCD radiative corrections still amount ${ }^{3}$ to $\sim 70 \%$. However, a large fraction of the corrections can be absorbed by choosing, for the proper renormalization scale, the BLM scale [17] which maps contributions associated with gluon self-energies into the effective QCD coupling; this is technically implemented by choosing $\mu$ such that the coefficient of the $N_{F}$ or $N_{L}$ term vanishes:

$$
\mu_{*}=e^{-\frac{7}{4}} M_{H} \approx 0.17 M_{H}
$$

The QCD corrections to the partial width

$$
\Gamma[H \rightarrow g g(g), q \bar{q} g]=\Gamma_{L O}\left[\alpha_{s}^{\left(N_{F}\right)}\left(\mu_{*}\right)\right]\left\{1+\frac{9}{2} \frac{\alpha_{s}^{\left(N_{F}\right)}\left(\mu_{*}\right)}{\pi}\right\}
$$

are reduced in this approach to a comfortable level of 15 to $25 \%$.

\subsection{Numerical evaluation}

The numerical analysis of the branching ratios for the Higgs decays in the Standard Model has been performed for the set of parameters given in the tables and the top quark massif

$$
M_{t}=(176 \pm 11) \mathrm{GeV}
$$

To estimate systematic uncertainties, the variation of the $c$ mass has been stretched over $2 \sigma$ and the uncertainty of the $b$ mass to $0.05 \mathrm{GeV}$. However, the dominant error in the predictions is due to the uncertainty in $\alpha_{s}$ which migrates to the running quark masses at the high energy scales.

The results for the branching ratios are displayed in Fig.2. Separately shown are the branching ratios for $\tau$ 's, $c, b$ quarks, gluons plus light quarks and electroweak gauge

\footnotetext{
${ }^{3}$ If the $b, c$ quarks are included in the final state, the partial width increases by an additional $20 \%$.

${ }^{4}$ This value of the top quark mass $M_{t}$ corresponds to the average of all measurements at the Tevatron presented in Ref. [18].
} 
bosons. The uncertainties in the prediction for the charm and gluon branching ratios are very large. Increasing $\alpha_{s}$ reduces the value of the running $c$ mass quite dramatically?.

Nevertheless, the expected hierarchy of the Higgs decay modes is clearly visible in Fig.2 despite these uncertainties. $B R_{\tau}$ is more than an order of magnitude smaller than $B R_{b}$, a straight consequence of the ratio between the two masses squared and the color factor. As a result of the small charm quark mass at the scale of the Higgs mass, the ratio of $B R_{c}$ to $B R_{b}$ is reduced by much more than an order of magnitude, which would have been naively expected. Taking these subtle QCD effects into account, the measurement of the decay branching ratios provides an excellent method to explore the physical nature of the Higgs particle.

\section{The Minimal Supersymmetric Standard Model}

We have performed a similar analysis for the hadronic decay modes of the Higgs bosons $h, H, A, H^{ \pm}$in the Minimal Supersymmetric extension of the Standard Model $(\mathcal{M S S M})$. Apart from the usual modifications $g_{Q}^{i}$ of the couplings, the analytic expressions for the partial widths of the scalar neutral Higgs bosons $h, H$ are the same as in the Standard Model, eqs.(3) and (7). In the massless quark limit [the general massive case is treated in the Appendix], the QCD radiatively corrected decay widths into quarks are given by

$$
\begin{gathered}
\Gamma[\Phi \rightarrow Q \bar{Q}]=\frac{3 G_{F} M_{\Phi}}{4 \sqrt{2} \pi} \bar{m}_{Q}^{2}\left(g_{Q}^{\Phi}\right)^{2}\left[\Delta_{\mathrm{QCD}}+\Delta_{t}^{\Phi}\right] \\
\Delta_{t}^{h / H}=\frac{g_{t}^{h / H}}{g_{Q}^{h / H}}\left(\frac{\alpha_{s}\left(M_{h / H}\right)}{\pi}\right)^{2}\left[1.57-\frac{2}{3} \log \frac{M_{h / H}^{2}}{M_{t}^{2}}+\frac{1}{9} \log ^{2} \frac{\bar{m}_{Q}^{2}\left(M_{h / H}\right)}{M_{h / H}^{2}}\right] \\
\Delta_{t}^{A}=\frac{g_{t}^{A}}{g_{Q}^{A}}\left(\frac{\alpha_{s}\left(M_{A}\right)}{\pi}\right)^{2}\left[3.83-\log \frac{M_{A}^{2}}{M_{t}^{2}}+\frac{1}{6} \log ^{2} \frac{\bar{m}_{Q}^{2}\left(M_{A}\right)}{M_{A}^{2}}\right] \\
\Gamma\left[H^{+} \rightarrow U \bar{D}\right]=\frac{3 G_{F} M_{H^{ \pm}}}{4 \sqrt{2} \pi}\left|V_{U D}\right|^{2}\left[\bar{m}_{U}^{2}\left(g_{U}^{A}\right)^{2}+\bar{m}_{D}^{2}\left(g_{D}^{A}\right)^{2}\right] \Delta_{\mathrm{QCD}}
\end{gathered}
$$

with $\Phi=h, H, A$. [Eq.(13) is valid if either the first or the second term is dominant.] The relative couplings $g_{Q}^{\Phi}$ have recently been collected in Ref.20; the masses in the Yukawa couplings are to be evaluated at the scales $M_{\Phi}$ and $M_{H^{ \pm}}$.

Since the $b$ quark couplings to the Higgs bosons may be strongly enhanced and the $t$ quark couplings suppressed in the $\mathcal{M S S M}, b$ loops can contribute significantly to the $g g$

\footnotetext{
${ }^{5}$ The value of $B R_{c}$ is significantly smaller in Ref. [7] for two reasons. (i) The ratio between the $\overline{\mathrm{MS}}$ mass $\bar{m}_{c}\left(M_{c}\right)$ and the pole mass $M_{c}^{p t 3}$ to $\mathcal{O}\left(\alpha_{s}^{2}\right)$ is smaller than the corresponding ratio for $M_{c}^{p t 2}$ to $\mathcal{O}\left(\alpha_{s}\right)$. However, since $\bar{m}_{c}\left(M_{c}\right)$ has been determined through QCD sum rules only to $\mathcal{O}\left(\alpha_{s}\right)$, the parameter $M_{c}=M_{c}^{p t 2}$ is the proper pole mass to be used in a consistent analysis up to $\mathcal{O}\left(\alpha_{s}\right)$. We have performed the evolution of the running $\overline{\mathrm{MS}}$ mass with and without the $\mathcal{O}\left(\alpha_{s}^{2}\right)$ contribution; the difference between the two results at the scale of the Higgs mass turned out to be negligible. The present analysis is therefore theoretically consistent. (ii) Moreover, in Ref. [7] the average LEP $\alpha_{s}$ value has been adopted which is larger than the world average value including deep-inelastic scattering data. This gives rise to a faster fall-off of the running charm mass $\sim\left[\alpha_{s}(\mu)\right]^{12 / 23}$ at large scales.
} 
coupling so that the approximation $M_{Q}^{2} \gg M_{H}^{2}$ cannot be applied any more in general. Nevertheless, it turns out a posteriori that this is an excellent approximation for the QCD corrections. The $L O$ width for $h, H \rightarrow g g$ is obtained from eq.(6) by substituting $A^{H} \rightarrow g_{Q}^{h, H} A^{h, H}$; for the pseudoscalar Higgs decays, we find [3]

$$
\begin{aligned}
\Gamma_{L O}[A \rightarrow g g] & =\frac{G_{F} \alpha_{s}^{2} M_{A}^{3} \mid}{16 \sqrt{2} \pi^{3}}\left|\sum_{t, b} g_{Q}^{A} A^{A}\left(\tau_{Q}\right)\right|^{2} \\
A^{A}(\tau) & =\tau f(\tau) \\
\Gamma[A \rightarrow g g(g), q \bar{q} g] & =\Gamma_{L O}\left[\alpha_{s}^{\left(N_{F}\right)}(\mu)\right]\left\{1+E^{N_{F}} \frac{\alpha_{s}^{\left(N_{F}\right)}(\mu)}{\pi}\right\} \\
E^{N_{F}} & =\frac{97}{4}-\frac{7}{6} N_{F}+\frac{33-2 N_{F}}{6} \log \frac{\mu^{2}}{M_{A}^{2}}
\end{aligned}
$$

To illustrate the size of the uncertainties introduced into the predictions by the QCD parameters, the branching ratios have been calculated for a specific set of parameters. The top mass is varied within $M_{t}=(176 \pm 11) \mathrm{GeV}$. In addition to the other parameters defined in the previous section, the running mass of the $s$ quark at the scale $1 \mathrm{GeV}$ and the CKM type mixing parameter $V_{c b}$ are chosen as

$$
\begin{aligned}
& \bar{m}_{s}(1 \mathrm{GeV})=(0.190 \pm 0.040) \mathrm{GeV} \\
& \left|V_{c b}\right|=0.040 \pm 0.008
\end{aligned}
$$

while the $\mathcal{S U S Y}$ parameters are set to

$$
\begin{aligned}
& \tan \beta=1.6 \\
& \mathcal{S U S Y} \text { scale parameter: } M_{S}=1 \mathrm{TeV} \\
& \text { "typical" mixing: } A_{t}=-\mu=1 \mathrm{TeV}
\end{aligned}
$$

$\mathcal{S U S Y}$ masses and couplings have been calculated according to the RG program described in Ref. 19]. Varying the $\mathcal{S U S Y}$ parameters does not change the picture of the QCD corrections and the uncertainties exemplified for the set of parameters chosen above. The final results 5 are displayed in Figs.3a-d. The branching ratios are separated again for final states including $b, c$ quarks [labeled $b \bar{b}$ and $c \bar{c}$ ] and gluons plus light quarks [labeled $g g]$. The main sources of uncertainties for the branching ratios are the charm and gluon decays. The branching ratios for $b$ and $\tau$ decays are less affected by $\alpha_{s}$. The uncertainty in the top quark mass affects primarily the upper limit of the light $\mathrm{CP}$-even scalar mass $M_{h}$ and the couplings at the electroweak level, as shown in Fig.3a. The uncertainty in $M_{h}$ migrates to the partial widths of the heavy Higgs bosons through cascade decays; this

\footnotetext{
${ }^{6}$ The Fortran code for the partial decay widths in the $\mathcal{S M}$ and $\mathcal{M S S M}$ may be obtained from djouadi@desy.de or spira@desy.de.
} 
second step is indicated by the error bars attached to the curves in Figs. 3b-d.

\section{Acknowledgements}

We are very grateful for discussions with A. Kataev, Y. Okada and M. Peskin. We thank M. Carena for providing us with the Fortran code for calculating the $\mathcal{S U S Y}$ Higgs parameters. A.D. thanks the Theory Group for the warm hospitality extended to him at DESY.

\section{APPENDIX}

For completeness, we present in this Appendix the expressions of the leading order QCD corrections to $\mathcal{S M}$ and $\mathcal{M S S M}$ Higgs boson decays involving non-zero mass effects for heavy quarks. As a general example we will consider top quarks.

The partial decay widths of the $\mathrm{CP}$-even Higgs bosons $\Phi=H_{\mathrm{SM}}, h$ and $H$ into top quark pairs, in terms of the top quark pole mass, is given by

$$
\Gamma[\Phi \rightarrow t \bar{t}]=\frac{3 G_{F} M_{\Phi}}{4 \sqrt{2} \pi}\left(g_{t}^{\Phi}\right)^{2} m_{t}^{2} \beta^{3}\left[1+\frac{4}{3} \frac{\alpha_{s}}{\pi} \Delta^{\Phi}\right]
$$

where $\beta=\left(1-4 m_{t}^{2} / M_{\Phi}^{2}\right)^{1 / 2}$ is the velocity of the top quarks. To leading order, the QCD correction factor is given by [8, 21, 22]

$$
\Delta^{\Phi}=\frac{1}{\beta} A(\beta)+\frac{1}{16 \beta^{3}}\left(3+34 \beta^{2}-13 \beta^{4}\right) \log \frac{1+\beta}{1-\beta}+\frac{3}{8 \beta^{2}}\left(7 \beta^{2}-1\right)
$$

with

$$
\begin{aligned}
A(\beta)= & \left(1+\beta^{2}\right)\left[4 \operatorname{Li}_{2}\left(\frac{1-\beta}{1+\beta}\right)+2 \operatorname{Li}_{2}\left(-\frac{1-\beta}{1+\beta}\right)-3 \log \frac{1+\beta}{1-\beta} \log \frac{2}{1+\beta}\right. \\
& \left.-2 \log \frac{1+\beta}{1-\beta} \log \beta\right]-3 \beta \log \frac{4}{1-\beta^{2}}-4 \beta \log \beta
\end{aligned}
$$

[ $\mathrm{Li}_{2}$ is the Spence function defined by $\operatorname{Li}_{2}(x)=-\int_{0}^{x} d y y^{-1} \log (1-y)$.]

The partial decay width of the $\mathrm{CP}$-odd Higgs boson $A$ into top quark pairs reads correspondingly

$$
\Gamma[A \rightarrow t \bar{t}]=\frac{3 G_{F} M_{A}}{4 \sqrt{2} \pi}\left(g_{t}^{A}\right)^{2} m_{t}^{2} \beta\left[1+\frac{4}{3} \frac{\alpha_{s}}{\pi} \Delta^{A}\right]
$$

where the QCD correction factor is given by [21, 22]

$$
\Delta^{A}=\frac{1}{\beta} A(\beta)+\frac{1}{16 \beta}\left(19+2 \beta^{2}+3 \beta^{4}\right) \log \frac{1+\beta}{1-\beta}+\frac{3}{8}\left(7-\beta^{2}\right)
$$


The partial decay width of the charged Higgs particles decay into top and bottom quarks may be written

$$
\begin{aligned}
\Gamma\left[H^{+} \rightarrow t \bar{b}\right]= & \frac{3 G_{F} M_{H^{ \pm}}}{4 \sqrt{2} \pi} \lambda^{1 / 2}\left\{( 1 - \mu _ { t } - \mu _ { b } ) \left[\frac{m_{t}^{2}}{\operatorname{tg}^{2} \beta}\left(1+\frac{4}{3} \frac{\alpha_{s}}{\pi} \Delta_{t b}^{+}\right)\right.\right. \\
& \left.\left.+m_{b}^{2} \operatorname{tg}^{2} \beta\left(1+\frac{4}{3} \frac{\alpha_{s}}{\pi} \Delta_{b t}^{+}\right)\right]-4 m_{t} m_{b} \sqrt{\mu_{t} \mu_{b}}\left(1+\frac{4}{3} \frac{\alpha_{s}}{\pi} \Delta_{t b}^{-}\right)\right\}
\end{aligned}
$$

where $\mu_{t}=m_{t}^{2} / M_{H^{ \pm}}^{2}, \mu_{b}=m_{b}^{2} / M_{H^{ \pm}}^{2}$ and $\lambda$ the usual two-body phase space function $\lambda=$ $\left(1-\mu_{t}-\mu_{b}\right)^{2}-4 \mu_{t} \mu_{b}$; again the quark masses are the pole masses. In the approximation where the $b$ quark mass is neglected the QCD factors $\Delta_{i j}^{ \pm}$are given by [22]

$$
\begin{aligned}
& \Delta_{i j}^{+}=\frac{9}{4}+\frac{3-2 \mu_{i}+2 \mu_{j}}{4} \log \frac{\mu_{i}}{\mu_{j}}+\frac{\left(\frac{3}{2}-\mu_{i}-\mu_{j}\right) \lambda+5 \mu_{i} \mu_{j}}{2 \lambda^{1 / 2}\left(1-\mu_{i}-\mu_{j}\right)} \log x_{i} x_{j}+B_{i j} \\
& \Delta_{i j}^{-}=3+\frac{\mu_{j}-\mu_{i}}{2} \log \frac{\mu_{i}}{\mu_{j}}+\frac{\lambda+2\left(1-\mu_{i}-\mu_{j}\right)}{2 \lambda^{1 / 2}} \log x_{i} x_{j}+B_{i j}
\end{aligned}
$$

with $x_{i}=2 \mu_{i} /\left[1-\mu_{i}-\mu_{j}+\lambda^{1 / 2}\right]$ and

$$
\begin{aligned}
B_{i j}= & \frac{1-\mu_{i}-\mu_{j}}{\lambda^{1 / 2}}\left[4 \operatorname{Li}_{2}\left(x_{i} x_{j}\right)-2 \operatorname{Li}_{2}\left(x_{i}\right)-2 \operatorname{Li}_{2}\left(x_{j}\right)+2 \log x_{i} x_{j} \log \left(1-x_{i} x_{j}\right)\right. \\
& -4\left[\log x_{i} \log \left(1-x_{i}\right)-\log x_{j} \log \left(1-x_{j} x_{j}\right)+\frac{x_{i} x_{j}}{1-x_{i} x_{j}} \log x_{i} x_{j}\right] \\
& +\frac{\lambda^{1 / 2}+\mu_{i}-\mu_{j}}{\lambda^{1 / 2}}\left[\log \left(1-x_{i}\right)+\frac{x_{i}}{1-x_{i}} \log x_{i}\right] \\
& +\frac{\lambda^{1 / 2}-\mu_{i}+\mu_{j}}{\lambda^{1 / 2}}\left[\log \left(1-x_{j}\right)+\frac{x_{j}}{1-x_{j}} \log x_{j}\right]
\end{aligned}
$$

Well above the $t \bar{t}$ threshold, the QCD factors $\Delta_{i j}^{+}$reduce to

$$
\begin{aligned}
\Delta_{t b}^{+} & =\frac{9}{4}+\frac{3}{2} \log \frac{m_{t}^{2}}{M_{H^{ \pm}}^{2}} \\
\Delta_{b t}^{+} & =\frac{9}{4}+\frac{3}{2} \log \frac{m_{b}^{2}}{M_{H^{ \pm}}^{2}}
\end{aligned}
$$

The large logarithms can be mapped into the running quark masses in the usual way. Adopting the $\overline{\mathrm{MS}}$ mass at the scale of the Higgs mass the bulk of the next-to-leading order correction is automatically included in this limit and the QCD corrections approach the common chirally invariant factor $\Delta_{\mathrm{QCD}}$, eq.(3)).

In the small mass limit, the non-logarithmic term of the factor $\Delta_{t b}^{-}$

$$
\Delta_{t b}^{-}=3+\frac{3}{2} \log \frac{m_{t}^{2}}{M_{H^{ \pm}}^{2}}+\frac{3}{2} \log \frac{m_{b}^{2}}{M_{H^{ \pm}}^{2}}
$$


differs from the corresponding term in $\Delta_{i j}^{+}(i, j=t, b)$, eq.(A.7). However, this is still in accordance with chiral symmetry since the correction eq. (A.8) is of subleading order in the small quark mass expansion.

\section{References}

[1] P.M. Zerwas, Proceedings of the International Conference on High Energy Physics, Marseille 1993; A. Djouadi, Int. Mod. Phys. A10 (1995) 1.

[2] H.M. Georgi, S.L. Glashow, M.E. Machacek and D.V Nanopoulos, Phys. Rev. Lett. 40 (1978) 692.

[3] M. Spira, A. Djouadi, D. Graudenz and P.M. Zerwas, Nucl. Phys. B453 (1995) 17.

[4] A. Djouadi, J. Kalinowski and P. M. Zerwas, Z. Phys. C54 (1992) 255.

[5] Z. Kunszt, Nucl. Phys. B247 (1984) 339; J.F. Gunion, Phys. Lett. B253 (1991) 269.

[6] M.D. Hildreth, T.L. Barklow and D.L. Burke, Phys. Rev. D49 (1994) 3441.

[7] E. Gross, B.A. Kniehl and G. Wolf, Z. Phys. C63 (1994) 417; (E) C66 (1995) 321.

[8] E. Braaten and J.P. Leveille, Phys. Rev. D22 (1980) 715; A.L. Kataev and V.T. Kim, Mod. Phys. Lett. A9 (1994) 1309; L.R. Surguladze, Phys. Lett. 341 (1994) 61.

[9] S.G. Gorishny, A.L. Kataev, S.A. Larin and L.R. Surguladze, Mod. Phys. Lett. A5 (1990) 2703; Phys. Rev. D43 (1991) 1633.

[10] K.G. Chetyrkin, J.H. Kühn and A. Kwiatkowski, Proceedings of the Workshop "QCD at LEP", Aachen 1994; K.G. Chetyrkin and A. Kwiatkowski, Report LBL-37269.

[11] J. Fleischer and F. Jegerlehner, Phys. Rev. D23 (1981) 2001; D.Yu. Bardin, B.M. Vilenskiu and P.Kh. Khristova, Sov. J. Nucl. Phys. 53 (1991) 152; A. Dabelstein and W. Hollik, Z. Phys. C53 (1992) 507; B.A. Kniehl, Nucl. Phys. B376 (1992) 3.

[12] N. Gray, D.J. Broadhurst, W. Grafe and K. Schilcher, Z. Phys. C48 (1990) 673.

[13] S. Narison, Phys. Lett. B341 (1994) 73.

[14] S. Bethke, Proceedings of the Workshop "QCD 94", Montpellier 1994.

[15] J. Ellis, M.K. Gaillard and D.V. Nanopoulos, Nucl. Phys. B106 (1976) 292. 
[16] T. Inami, T. Kubota and Y. Okada, Z. Phys. C18 (1983) 69; A. Djouadi, M. Spira and P.M. Zerwas, Phys. Lett. B264 (1991) 440.

[17] S.J. Brodsky, G.Peter Lepage and P.B. Mackenzie, Phys. Rev. D28 (1983) 228.

[18] A. Menzione, Proceedings, International Conference on High Energy Physics, Brussels 1995 .

[19] M. Carena, J. Espinosa, M. Quiros and C. Wagner, Phys. Lett. B355 (1995) 209.

[20] A. Djouadi, J. Kalinowski and P. M. Zerwas, Report DESY 95-211.

[21] M. Drees and K. Hikasa, Phys. Lett. B240 (1990) 455.

[22] A. Djouadi and P. Gambino, Phys. Rev. D51 (1995) 218.

\section{Figure Captions}

Fig. 1: (a) Generic Feynman diagrams for the decay processes $H \rightarrow b \bar{b}, c \bar{c}$ and $H \rightarrow$ $b \bar{b} g, c \bar{c} g$. (b) Feynman diagrams for $H \rightarrow g g$ decays and final-state gluon splitting into quarks.

Fig. 2: Branching ratios of the $\mathcal{S} \mathcal{M}$ Higgs boson including the uncertainties from the quark masses and the QCD coupling $\alpha_{s}$. The resulting errors of the branching ratios are presented as shaded bands. The curves labeled $b \bar{b}$ and $c \bar{c}$ are the inclusive decay widths; they account for all final states including $c$ and $b$ quarks. The curve labeled $g g$ corresponds accordingly to gluon and light-quark final states only.

Fig. 3: Branching ratios of the $\mathcal{M S S M}$ Higgs bosons $h, H, A, H^{ \pm}[(\mathrm{a}) . .(\mathrm{d})]$, including uncertainties from the quark masses $m_{b}, m_{c}, m_{s}$ and the strong coupling $\alpha_{s}$. In the widths, the three-body channels [20] have been included. The top quark mass is fixed at $M_{t}=$ $176 \mathrm{GeV}$ for the shaded error bands and the error bars shown below the $200 \mathrm{GeV}$ mark in figure (b). The additional uncertainty due to the top mass is marked by error bars in the figures (b) [at $M_{H}>200 \mathrm{GeV}$ ], (c) and (d). In figure (a) the curves for the upper and lower limit of the top mass band are presented separately, using the average values of the other quark masses and of the strong coupling $\alpha_{s}$ for the sake of clarity. The labels follow the definitions in Fig.2; i.e. the branching ratios are classified according to the inclusive hadronic final states with [labels $b \bar{b}, c \bar{c}$ ] and without heavy quarks [label $g g$ ]. 
(a)

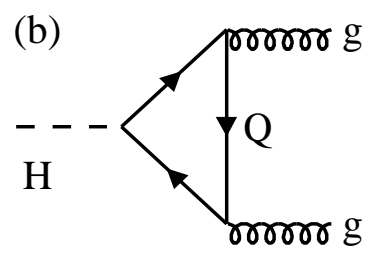

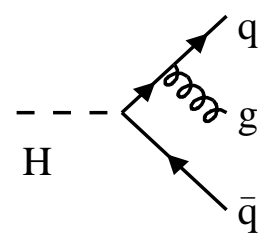

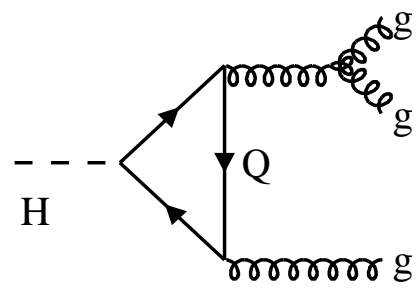

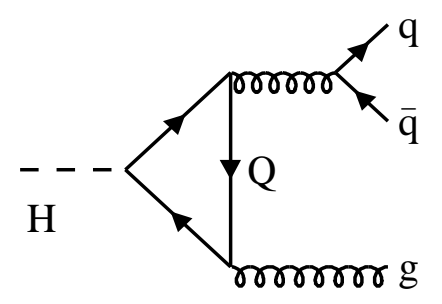

Fig. 1

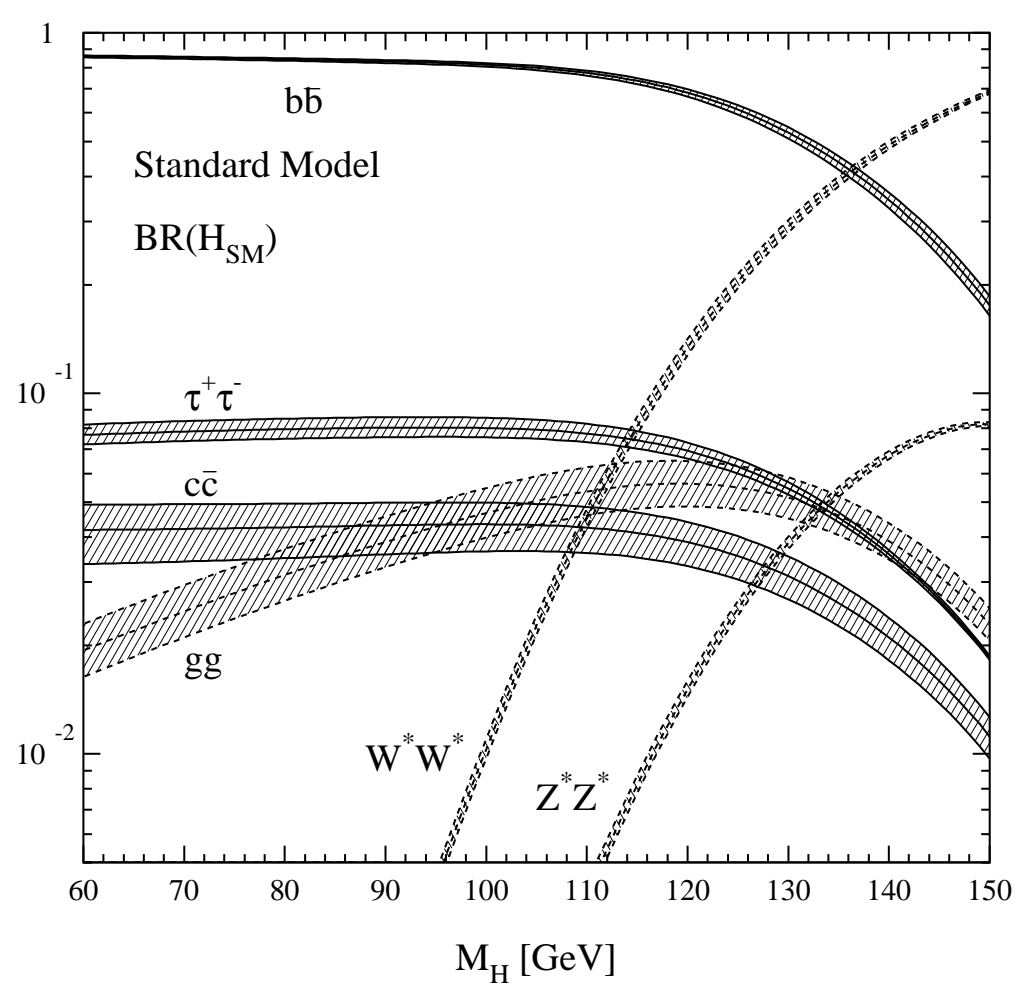

Fig. 2 


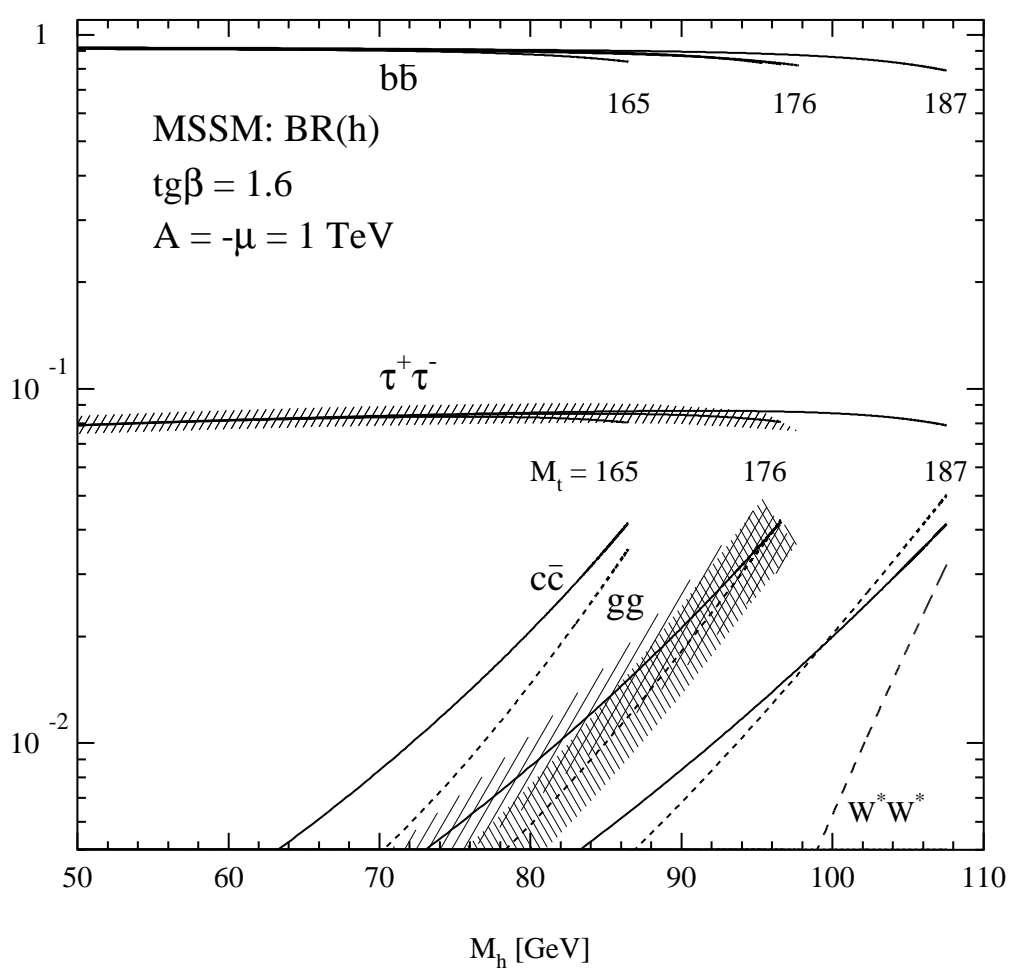

Fig. 3a

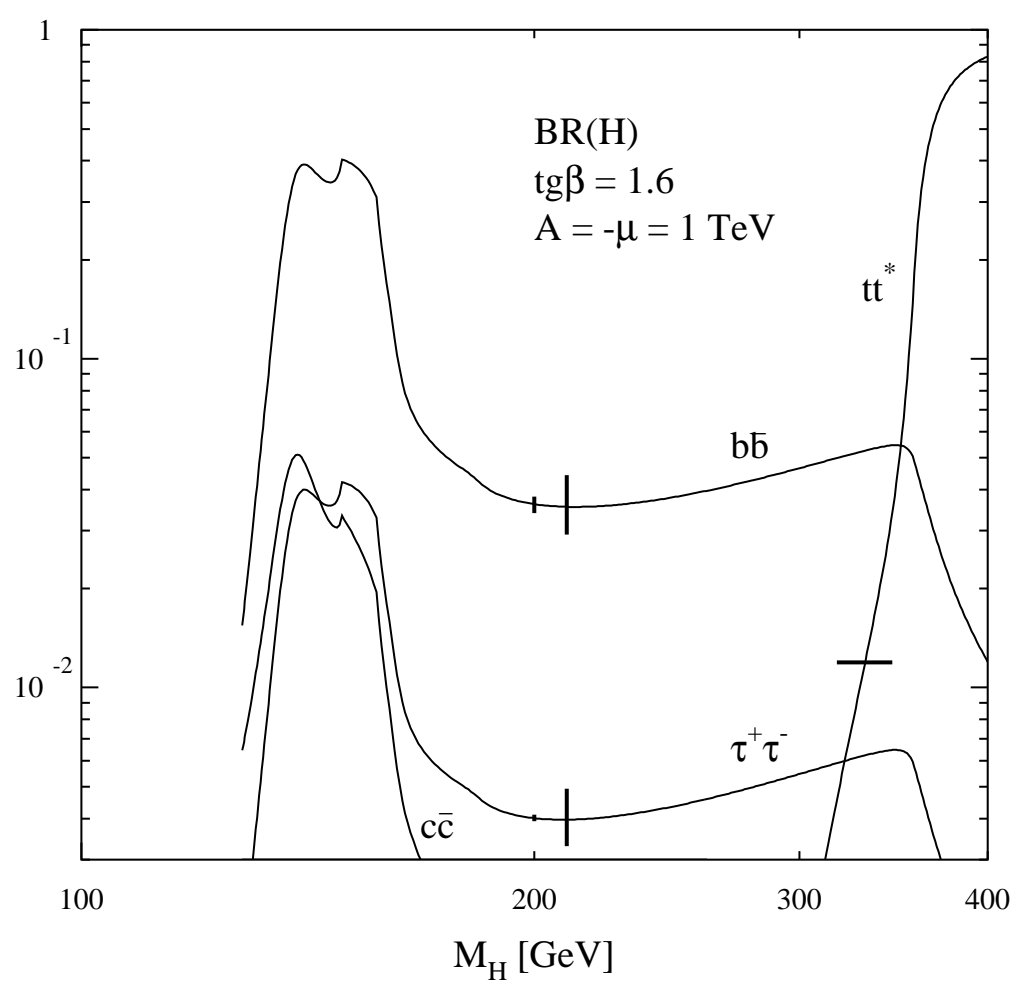

Fig. 3b 

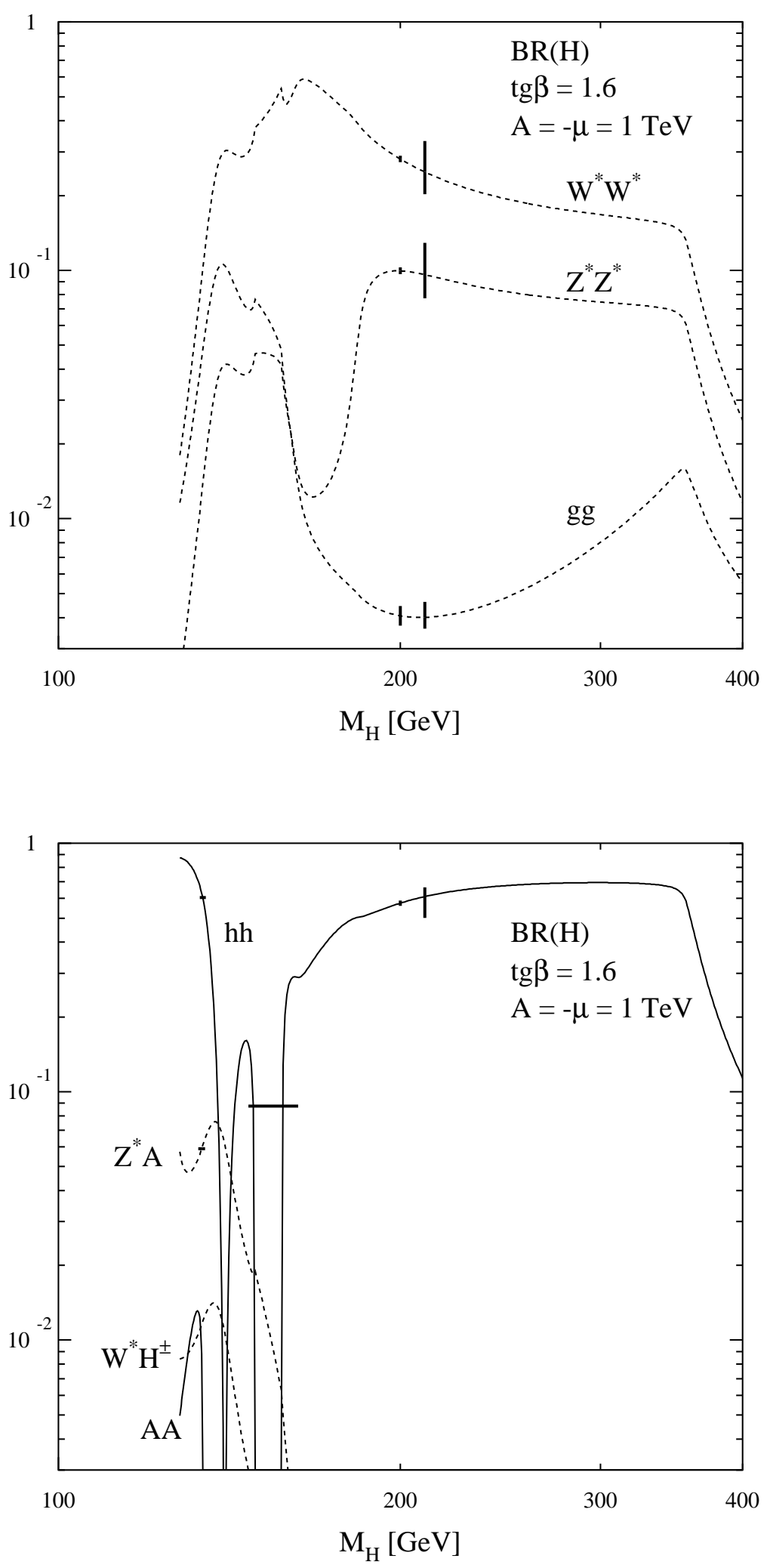

Fig. 3b cont'd 


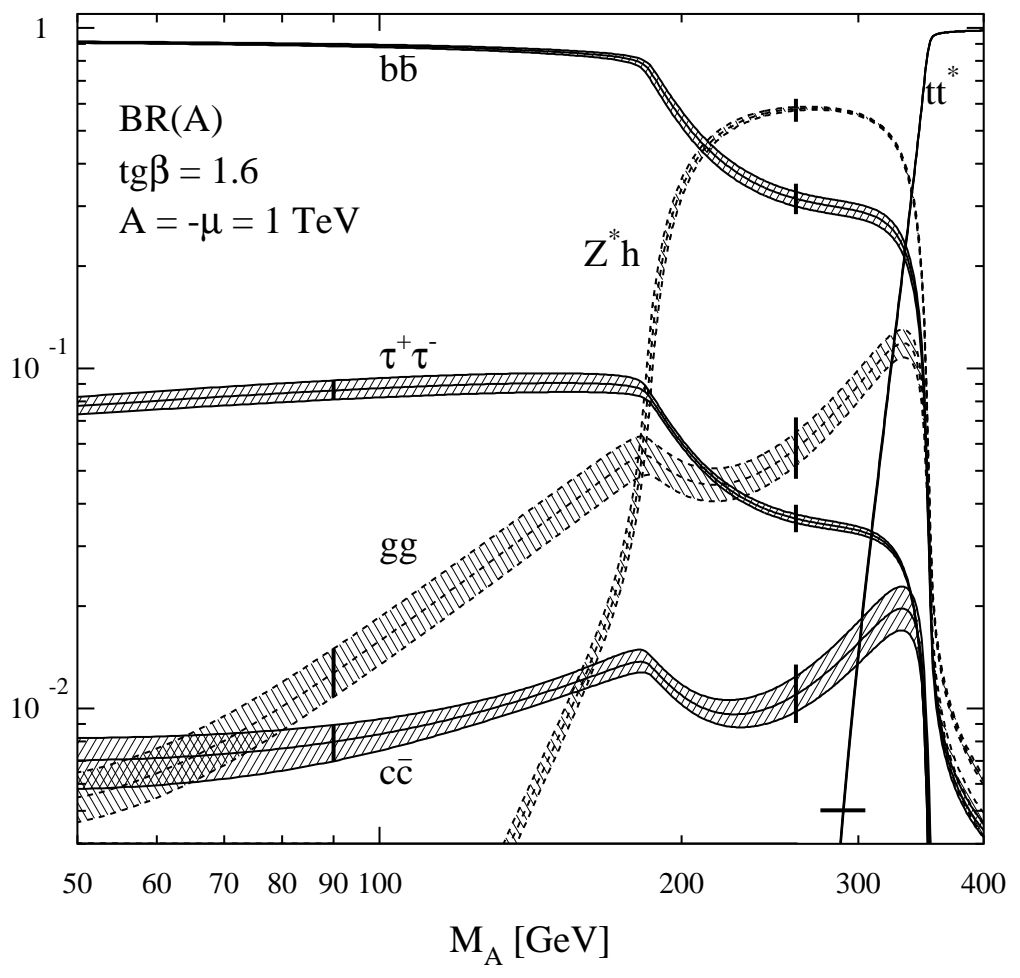

Fig. 3c

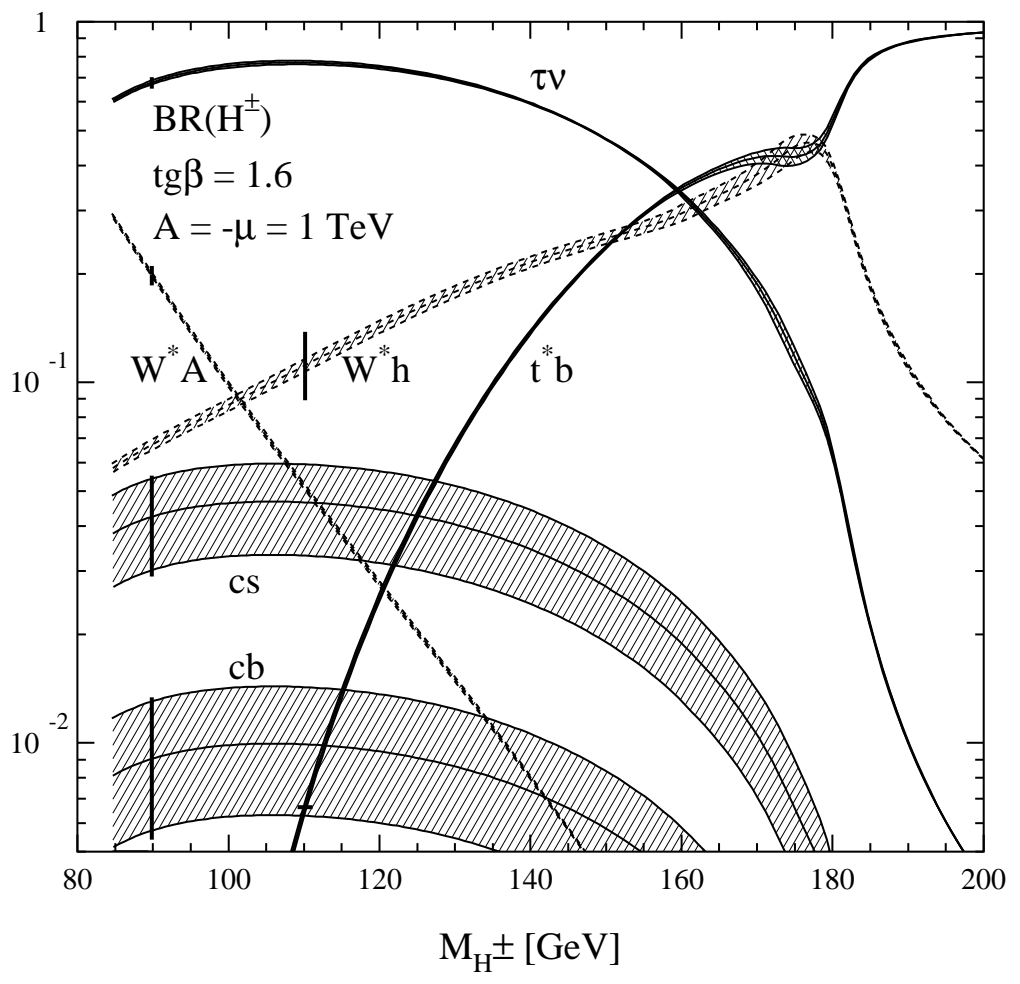

Fig. 3d 